\title{
Effect of Chemical Reaction towards MHD Marginal Layer Movement of Casson Nanofluid through Porous Media above a Moving Plate with an Adaptable Thickness
}

\section{Ganugapati Raghavendra Ganesh and Wuriti Sridhar*}

Department of Mathematics, Koneru Lakshmaiah Education Foundation, Guntur-522502, Andhra Pradesh, India

\begin{abstract}
In the current workflow and heat exchange of a Casson nanoliquid across a penetrable media above a moving plate with variable thermal conductivity, adaptive thickness and chemical reaction are analyzed. First, the governing nonlinear equations of partial derivative terms with proper extreme conditions are changed into equations of ordinary derivative terms with suitable similarity conversions. Then the resulting equations are worked out using the Keller box method. The effects of various appropriate parameters are analyzed by constructing the visual representations of velocity, thermal, and fluid concentration. The velocity profile increased for shape parameter, and the opposite trend is observed for magnetic, Casson, porosity parameters. Temperature profile increases for magnetic, Casson, Brownian motion parameter, and thermophoresis parameters. Concentration profiles show a decreasing trend for wall thickness, Brownian movement, chemical reaction parameters. Also, skin friction values and calculated and matched with previous literature found in accordance. Also, local parameters Nusselt and Sherwood numbers are calculated and analyzed in detail.
\end{abstract}

Keywords: Adaptive thickness, Casson nanofluid, chemical reaction, porous medium, thermal conductivity, thermal radiation

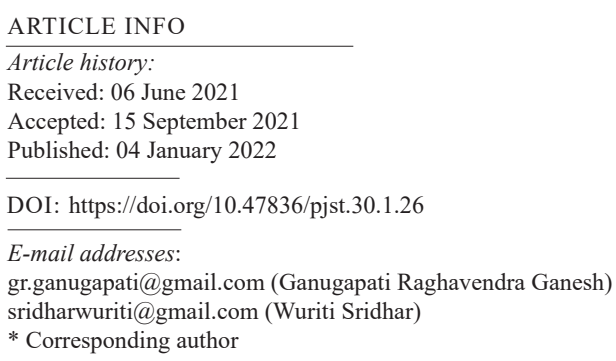

\section{INTRODUCTION}

Heat transfer plays a very significant role in many industrial processes. Naturally, the thermal conductivity of traditional fluids is very low. So, to develop the thermal properties of the fluid dispersing nanometer-sized particles in base fluids, a new pioneering class of fluids, which has 
outstanding thermal properties and conserving capability, are generated entitled nanofluids. Also, the usage of nanofluids has various applications thermal management of industrial and consumer products. By collaborating nanoparticles in the fluids may reduce erosion and clogging in systems, for long term reliability of the industrial equipment, efficient cooling is most important, so it has application in tribological and biomedical applications as described by Das et al. (2007). Choi (2008) described that nanofluids have very prospective applications in the areas of microelectronics, space, biomedical industries, and defense. Wang and Mujumdar (2008a) go through the recent findings in using nanofluids and their applications and conclude that communication between particle and liquid should show a vital role in the convective heat transferal performance of fluids. Later, Wang and Mujumdar (2008b) reviewed the application of nanofluids and observed a specific application in the biomedical industry like usage in cancer treatment methods to kill tumor cells effectively, safer surgery processes to reduce organ damages. After that, Singh (2008) focuses on the studies related to the thermal conductivity of nanofluids.

Later, Beg et al. (2009) studied nanofluid flow on an oscillating inclined plate with MHD effect concluded that an upsurge in Hartmann magnetic parameter drops velocity and enhances the thickness of the momentum boundary layer. Fang et al. (2012) investigate the momentum boundary layer with non-uniform thickness observes that if the velocity power index is below one, the non-horizontalness presents a mass suction effect, whereas the velocity power index is above one, the non-horizontalness causes an impact of mass injection. Sheikholeslami et al. (2014) discussed 3D nanofluid movement in the middle of two parallel revolving flat surfaces and concluded the degree of the skin friction coefficient increasing function of the magnetic parameter. Malik et al. (2014) describe Casson nanofluid flow on a vertical cylinder using the RK-Fehlberg method. Mahdy and Chamka (2015) take on Buongiorno's model to study Casson nanofluid flow and observe that Casson parameter restrain the velocity field. Besthapu and Bandari (2015) studied Casson nanofluid flow on a nonlinear elongated sheet concluded that enhancement in Grashoff number reduces the temperature of the fluid. Uddin et al. (2015) described Casson fluid flow with slip parameter and observed that increasing thermal slip nanoparticles concentration would be enhanced. Abdel-wahed et al. (2015) observed that the nanosized speck concentration close to the non-flat surface is superior and narrow than on the flat surface. Oyelakin et al. (2016) studied fluid flow with Soret and Dofour effects using the spectral relaxation method. After that, Ibrahim and Makinde (2016) analyzed the stagnation point Casson nanofluid stream and concluded that for growth in Casson and magnetic parameters, thermal boundary thickness enhanced. After that, Ahmad et al. (2017) noted that on increasing Brownian motion parameters, surface temperature rises. Reddy et al. (2017) used the SQLM technique to study Williamson nanofluid flow on the elongated sheet with adaptable thickness concludes for progressive values of Williamson parameter 
concentration of fluid enhances. Dharmaiah et al. (2017) considered nanofluid flow on a vertical flat plate and remarked that heat transfer rate would be enhanced by an escalation of heat source parameters. Madaki et al. (2017) examine nanofluid flow on the elongated sheet with radiation and chemical reaction effects using the OHAM technique, and the RK fourth-order method observes that increasing radiation parameter thermal boundary layer thickness increases. Later Biswas and Ahmad (2018) considered the Casson nanofluid flow with hall current and chemical reaction effects observed that increasing magnetic parameter velocity profiles reduces. Mahmood et al. (2018) studied the transfer of the heat and entropy implications on Casson nanofluid flow employing the Cattaneo-Christov model using a finite difference scheme. Later Reddy et al. (2018) considered the slipstream of a Newtonian nanofluid with adaptive surface thickness using RK with shooting technique observes heat transfer ability of various base fluids. Konda et al. (2018) studied mixed convection Casson nanofluid stream observes concentration outline falls with several values of chemical response parameter. Talla et al. (2018) considered Casson fluid flow on exponentially elongated sheet concludes concentration rises for growing values of Casson parameter. Khan et al. (2018) employed the bvp4c procedure to study Casson nanoliquid flow on the elongated surface with adaptive thickness concluded coefficient of skin friction will be diminished for enhancement of Casson parameter. Dharmaiah et al. (2019b) used the perturbation method to study rotating frame nanofluid flow and noted that chemical reaction parameter controls the concentration profiles. Later Dharmaiah et al. (2019a) examined viscous dissipation using the perturbation effect, and it was found that the skin friction drag augmented in view of reduction in the concentration buoyancy effects. Dharmaiah et al. (2020) analyzed hall and slip effects in magneto titanium alloy nanofluid concluded that velocity profiles increase increasing values of hall and ion slip parameters. Vijaya et al. (2020) studied liquid thin film flow of Casson nanofluid flow remarks that temperature profile escalates for higher values of magnetic, Casson parameters. Nagalakshm and Vijaya (2020) used R with bvpsolver to study the Carreau nanofluid flow on a nonlinearly stretching sheet. Sridhar and Lakshmi (2020) discussed nanofluid flow on elongating sheet through the double stratified medium using implicit finite difference method concludes that velocity slip parameter reduces concentration profile. Finally, Ismail et al. (2021) analyzed Casson nanofluid flow with variable thermal conductivity, and variable thickness of the fluids concluded that using Casson nanofluid will increase the movement of heat value over the exterior, which will accelerate the cooling process, and this, in turn, leads to improve the heat treatment process. Also, Casson nanofluid increases the shear stress above the surface and the level of mass relocation over the surface. The present work examined the Casson nanofluid flow on a moving surface with adaptable thermal conductivity and irregular thickness with porous media and chemical reaction effects by using Keller box method. 


\section{METHODOLOGY}

\section{Formulation of the Problem}

In the present study, two-dimensional Casson nanofluid flow with adaptive thermal conductivity near a moveable surface with irregular thickness is considered. The thickness of the surface is given by $y=\zeta(x+b)^{-\left(\frac{n-1}{2}\right)}$.Here, $\zeta$ is small. The plate is movable with irregular velocity $u_{w}{ }^{*}=a\{x+b\}^{m}$. The boundary layer is influenced by nonlinear heat generation, thermal radiation and nonlinear transverse magnetic field, chemical reaction effects. Here the moving plate is considered in the path of the x-axis, and the y-axis is upright to the surface. The governing partial differential equations corresponding to 2 dimensional Casson nanofluid stream on a movable surface with radiation, porous mediumplus chemical reaction effects with adaptive thermal conductivity are as in Equations 1-4:

$$
\begin{aligned}
& \frac{\partial u}{\partial x}+\frac{\partial v}{\partial y}=0 \\
& u \frac{\partial u}{\partial x}+v \frac{\partial u}{\partial y}=\frac{v}{\beta} \frac{\partial^{2} u}{\partial y^{2}}+v \frac{\partial^{2} u}{\partial y^{2}}-\frac{\sigma B^{2}}{\rho} u-\frac{v}{k} u \\
& u \frac{\partial T}{\partial x}+v \frac{\partial T}{\partial y}=\frac{1}{\rho c_{p}} \frac{\partial}{\partial y}\left(k \frac{\partial T}{\partial y}\right)-\frac{1}{\rho c_{p}} \frac{\partial q_{r}}{\partial y}+\tau\left[D_{b} \frac{\partial T}{\partial y} \frac{\partial C}{\partial y}+\frac{D_{t}}{T_{\infty}}\left(\frac{\partial T}{\partial y}\right)^{2}\right]+\frac{Q}{\rho c_{p}}\left(T-T_{\infty}\right) \\
& u \frac{\partial C}{\partial x}+v \frac{\partial C}{\partial y}=D_{b}\left(\frac{\partial^{2} C}{\partial y^{2}}\right)+\frac{D_{t}}{T_{\infty}}\left(\frac{\partial^{2} T}{\partial y^{2}}\right)-k^{*}\left(C-C_{\infty}\right)
\end{aligned}
$$

Magnetic field strength $B=B_{0}(x+b)^{\frac{m-1}{2}}$ and heat generation $Q=Q_{0}(x+b)^{m-1}$, and thermal conductivity $\mathrm{k}$ are assumed to be $k=k_{\infty}\left(1+\frac{\varepsilon\left(T-T_{\infty}\right)}{T_{w}-T_{\infty}}\right)$. Flow-through

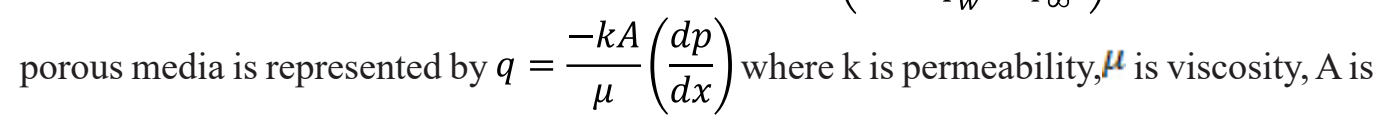
the area of a cross-section through porous media.

Constraint set for boundary is (Equation 5):

$$
\begin{aligned}
& u=u_{w}{ }^{*}, v=0, T=T_{w}{ }^{*}, C=C_{w}{ }^{*} \text { at } y=(x+b)^{\frac{1-m}{2}} \\
& u=0, T=T_{\infty}, C=C_{\infty} \text { at } y \rightarrow \infty
\end{aligned}
$$

Similarity transformations (Reddy et al., 2017) used are as in Equation 6 


$$
\left.\begin{array}{rl}
\eta & =\sqrt{\frac{a}{v} \frac{(m+1)}{2}}\left[(x+b)^{\frac{m-1}{2}} y-A\right] \\
\varphi & =\sqrt{\frac{2}{m+1} a v(x+b)^{\frac{m+1}{2}} f} \\
T & =T_{\infty}+\theta(\eta)\left(T_{w}{ }^{*}-T_{\infty}\right) \\
C & =C_{\infty}+\phi(\eta)\left(C_{w}{ }^{*}-C_{\infty}\right)
\end{array}\right\}
$$

Using similarity transforms governing Equations 2-4 are transformed to Equations 7-9:

$$
\begin{aligned}
& \left(1+\frac{1}{\beta}\right) f^{\prime \prime \prime}+f f^{\prime \prime}-\frac{2 m}{m+1} f^{\prime 2}-\frac{2 M}{m+1} f^{\prime}-\frac{2 K_{1}}{m+1} f^{\prime}=0 \\
& \left(\frac{3+4 R d}{3 \operatorname{Pr}}\right)\left[\theta^{\prime \prime}+\varepsilon \theta \theta^{\prime \prime}+\varepsilon \theta^{\prime 2}\right]+f \theta^{\prime}+N b \theta^{\prime} \phi^{\prime}+N t \theta^{\prime 2}+\left(\frac{2 \lambda}{m+1}\right) \theta=0 \\
& \phi^{\prime \prime}+\text { Lef } \phi^{\prime}+\frac{N t}{N b} \theta^{\prime \prime}-S c \gamma \phi=0
\end{aligned}
$$

Also, boundary conditions (Equation 5) are transformed into Equation 10

$$
\begin{aligned}
& f(0)=\frac{1-m}{1+m} \alpha, f^{\prime}(0)=1, \theta(0)=1, \phi(0)=1 \\
& f^{\prime}(\infty)=0, \theta(\infty)=0, \phi(\infty)=0
\end{aligned}
$$

\section{Numerical Scheme}

Equations 7-9 subject to boundary condition (Equation 10) are solved using the Keller box method, mentioned as in the flow chart. In solving a parabolic system of partial differential equations, and Newton's method is implemented to linearize the system, an adequate initial guess is chosen to get the required solution.

Introducing Equation 11:

$$
f^{\prime}=p, p^{\prime}=q, g=\theta, g^{\prime}=t, s=\phi, s^{\prime}=n
$$

Equations 7-9 are converted to the form Equations 12-14:

$$
\begin{aligned}
& \left(1+\frac{1}{\beta}\right) q^{\prime}+f q-\frac{2 m}{m+1} p^{2}-\frac{2 M}{m+1} p-\frac{2 K_{1}}{m+1} p=0 \\
& \left(\frac{3+4 R d}{3 \operatorname{Pr}}\right)\left[t^{\prime}+\varepsilon g t^{\prime}+\varepsilon t^{2}\right]+f t+N b t n+N t t^{2}+\left(\frac{2 \lambda}{m+1}\right) g=0
\end{aligned}
$$


$n^{\prime}+L e f n+\frac{N t}{N b} t^{\prime}-S c \gamma s=0$

Central differences are introduced, and Newton's method is used to get the system of linear Equations 15-50:

$\delta f_{J}-\delta f_{J-1}-\frac{h_{j}}{2}\left(\delta p_{J}\right)-\frac{h_{j}}{2}\left(\delta p_{J-1}\right)=\left(r_{1}\right)_{J}$

$\delta p_{J}-\delta p_{J-1}-\frac{h_{J}}{2}\left(\delta q_{J}\right)-\frac{h_{J}}{2}\left(\delta q_{J-1}\right)=\left(r_{2}\right)_{J}$

$\delta g_{J}-\delta g_{J-1}-\frac{h_{J}}{2}\left(\delta t_{J}\right)-\frac{h_{J}}{2}\left(\delta t_{J-1}\right)=\left(r_{3}\right)_{J}$

$\delta s_{J}-\delta s_{J-1}-\frac{h_{J}}{2}\left(\delta n_{J}\right)-\frac{h_{J}}{2}\left(\delta n_{J-1}\right)=\left(r_{4}\right)_{J}$

$\left(a_{1}\right)_{J} \delta q_{J}+\left(a_{2}\right)_{J} \delta q_{J-1}+\left(a_{3}\right)_{J} \delta f_{J}+\left(a_{4}\right)_{J} \delta f_{J-1}+\left(a_{5}\right)_{J} \delta p_{J}+\left(a_{6}\right)_{J} \delta p_{J-1}=\left(r_{5}\right)_{J}$

$\left(b_{1}\right)_{J} \delta t_{J}+\left(b_{2}\right)_{J} \delta t_{J-1}+\left(b_{3}\right)_{J} \delta g_{J}+\left(b_{4}\right)_{J} \delta g_{J-1}+\left(b_{5}\right)_{J} \delta f_{J}+\left(b_{6}\right)_{J} \delta f_{J-1}$

$+\left(b_{7}\right)_{J} \delta n_{J}+\left(b_{8}\right)_{J} \delta n_{J-1}=\left(r_{6}\right)_{J}$

$\left(c_{1}\right)_{J} \delta n_{J}+\left(c_{2}\right)_{J} \delta n_{J-1}+\left(c_{3}\right)_{J} \delta f_{J}+\left(c_{4}\right)_{J} \delta f_{J-1}+\left(c_{5}\right)_{J} \delta t_{J}+\left(c_{6}\right)_{J} \delta t_{J-1}$

$+\left(c_{7}\right)_{J} \delta s_{J}+\left(c_{8}\right)_{J} \delta s_{J-1}=\left(r_{7}\right)_{J}$

where

$$
\begin{aligned}
& \left(a_{1}\right)_{J}=1+\frac{\beta h_{J}}{4(\beta+1)}\left(f_{J}+f_{J-1}\right) \\
& \left(a_{2}\right)_{J}=-1+\frac{\beta h_{J}}{4(\beta+1)}\left(f_{J}+f_{J-1}\right) \\
& \left(a_{3}\right)_{J}=\frac{\beta h_{J}}{4(\beta+1)}\left(q_{J}+q_{J-1}\right) \\
& \left(a_{4}\right)_{J}=\left(a_{3}\right)_{J} \\
& \left(a_{5}\right)_{J}=\frac{-m \beta h_{J}}{(m+1)(\beta+1)}\left(p_{J}+p_{J-1}\right)-\frac{M \beta h_{J}}{(m+1)(\beta+1)}-\frac{K \beta h_{J}}{(m+1)(\beta+1)} \\
& \left(a_{6}\right)_{J}=\frac{-m \beta h_{J}}{(m+1)(\beta+1)}\left(p_{J}+p_{J-1}\right)-\frac{M \beta h_{J}}{(m+1)(\beta+1)}-\frac{K \beta h_{J}}{(m+1)(\beta+1)}
\end{aligned}
$$




$$
\begin{aligned}
& \left(b_{1}\right)_{J}=1+\frac{\varepsilon}{2}\left(g_{J}+g_{J-1}\right)+\frac{\varepsilon h_{J}}{2}\left(t_{J}+t_{J-1}\right)+\frac{3 \operatorname{Pr} h_{J}}{4(3+4 R d)}\left(f_{J}+f_{J-1}\right) \\
& +\frac{3 \operatorname{Pr} N b h_{J}}{4(3+4 R d)}\left(n_{J}+n_{J-1}\right)+\frac{3 \operatorname{Pr} N t h_{J}}{2(3+4 R d)}\left(t_{J}+t_{J-1}\right)
\end{aligned}
$$

$\left(b_{2}\right)_{J}=-1-\frac{\varepsilon}{2}\left(g_{J}+g_{J-1}\right)+\frac{\varepsilon h_{J}}{2}\left(t_{J}+t_{J-1}\right)+\frac{3 \operatorname{Pr} h_{J}}{4(3+4 R d)}\left(f_{J}+f_{J-1}\right)$

$+\frac{3 \operatorname{Pr} N b h_{J}}{4(3+4 R d)}\left(n_{J}+n_{J-1}\right)+\frac{3 \operatorname{Pr} N t h_{J}}{2(3+4 R d)}\left(t_{J}+t_{J-1}\right)$

$$
\left(b_{3}\right)_{j}=\frac{\varepsilon}{2}\left(t_{J}-t_{J-1}\right)+\frac{3 \operatorname{Pr} \lambda h_{J}}{(n+1)(3+4 R d)}
$$

$\left(b_{4}\right)_{J}=\left(b_{3}\right)_{J}$

$$
\left(b_{5}\right)_{J}=\frac{3 \operatorname{Pr} h_{J}}{4(3+4 R d)}\left(t_{J}+t_{J-1}\right)
$$

$\left(b_{6}\right)_{J}=\left(b_{5}\right)_{J}$

$\left(b_{7}\right)_{J}=\frac{3 \operatorname{Pr} N b h_{J}}{4(3+4 R d)}\left(t_{J}+t_{J-1}\right)$

$\left(b_{8}\right)_{J}=\left(b_{7}\right)_{J}$

$\left(c_{1}\right)_{J}=1+\frac{\operatorname{Leh}_{J}}{4}\left(f_{J}+f_{J-1}\right)$

$\left(c_{2}\right)_{J}=-1+\frac{\text { Leh }_{J}}{4}\left(f_{J}+f_{J-1}\right)$

$\left(c_{3}\right)_{J}=\frac{\operatorname{Leh}_{J}}{4}\left(n_{j}+n_{J-1}\right)$

$\left(c_{4}\right)_{J}=\left(c_{3}\right)_{J}$

$\left(c_{5}\right)_{J}=\frac{N t}{N b}$

$\left(c_{6}\right)_{J}=-\frac{N t}{N b}$

$\left(c_{7}\right)_{J}=-\frac{S c \gamma h_{J}}{2}$ 


$$
\begin{aligned}
& \left(c_{8}\right)_{J}=-\frac{S c \gamma h_{J}}{2} \\
& \left(r_{1}\right)_{J}=f_{J-1}-f_{J}+\left(\frac{h_{J}}{2}\right)\left(p_{J}+p_{J-1}\right) \\
& \left(r_{2}\right)_{J}=p_{J-1}-p_{J}+\left(\frac{h_{J}}{2}\right)\left(q_{J}+q_{J-1}\right) \\
& \left(r_{3}\right)_{J}=g_{J-1}-g_{J}+\left(\frac{h_{J}}{2}\right)\left(t_{J}+t_{J-1}\right) \\
& \left(r_{4}\right)_{J}=s_{J-1}-s_{J}+\left(\frac{h_{J}}{2}\right)\left(n_{J}+n_{J-1}\right) \\
& \left(r_{5}\right)_{J}=q_{J-1}-q_{J}-\frac{\beta h_{J}}{4(\beta+1)}\left(f_{J}+f_{J-1}\right)\left(q_{J}+q_{J-1}\right)+\frac{2 m \beta h_{J}}{4(m+1)(\beta+1)}\left(p_{J}+p_{J-1}\right)^{2} \\
& +\frac{2 M \beta h_{J}}{2(m+1)(\beta+1)}\left(p_{J}+p_{J-1}\right)+\frac{2 K \beta h_{J}}{2(m+1)(\beta+1)}\left(p_{J}+p_{J-1}\right) \\
& \left(r_{6}\right)_{J}=t_{J-1}-t_{J}-\frac{\varepsilon}{2}\left(g_{J}+g_{J-1}\right)\left(t_{J}-t_{J-1}\right)-\frac{\varepsilon h_{J}}{4}\left(t_{J}+t_{J-1}\right)^{2} \\
& -\frac{3 \operatorname{Pr} \lambda h_{J}}{(m+1)(3+4 R d)}\left(g_{J}+g_{J-1}\right)-\frac{3 \operatorname{Pr} h_{J}}{4(3+4 R d)}\left(f_{J}+f_{J-1}\right)\left(t_{J}+t_{J-1}\right) \\
& -\frac{3 \operatorname{Pr} N b h_{J}}{4(3+4 R d)}\left(n_{J}+n_{J-1}\right)\left(t_{J}+t_{J-1}\right)-\frac{3 \operatorname{Pr} N t h_{J}}{4(3+4 R d)}\left(t_{J}+t_{J-1}\right)^{2} \\
& \left(r_{7}\right)_{J}=n_{J-1}-n_{J}-\frac{L e \operatorname{Pr} h_{J}}{4}\left(f_{J}+f_{J-1}\right)\left(n_{J}+n_{J-1}\right)-\frac{N t}{N b}\left(t_{J}-t_{J-1}\right)+\frac{S c r h_{J}}{2}\left(s_{J}+s_{J-1}\right)
\end{aligned}
$$

The above linear set of simultaneous will be converted matrix notation (Equation 51). Taking $\mathrm{J}=1,2,3 \ldots \ldots$

$$
\left.\begin{array}{l}
\left\{A_{1}\right\}\left\{\delta_{1}\right\}+\left\{C_{1}\right\}\left\{\delta_{2}\right\}=\left\{r_{1}\right\} \\
\left\{B_{2}\right\}\left\{\delta_{1}\right\}+\left\{A_{2}\right\}\left\{\delta_{2}\right\}+\left\{C_{2}\right\}\left\{\delta_{3}\right\}=\left\{r_{2}\right\} \\
----------------- \\
----\left\{B_{j-1}\right\}\left\{\delta_{1}\right\}+\left\{A_{j-1}\right\}\left\{\delta_{2}\right\}+\left\{C_{j-1}\right\}\left\{\delta_{3}\right\}=\left\{r_{j-1}\right\} \\
------------\left\{B_{j}\right\}\left\{\delta_{j-1}\right\}+\left\{A_{j}\right\}\left\{\delta_{j}\right\}=\left\{r_{j}\right\}
\end{array}\right\}
$$


Where

$$
\begin{aligned}
& A_{1}=\left[\begin{array}{ccccccc}
0 & 0 & 0 & 1 & 0 & 0 & 0 \\
d & 0 & 0 & 0 & d & 0 & 0 \\
0 & d & 0 & 0 & 0 & d & 0 \\
0 & 0 & -1 & 0 & 0 & 0 & d \\
{\left[a_{2}\right]_{1}} & 0 & 0 & {\left[a_{3}\right]_{1}} & {\left[a_{1}\right]_{1}} & 0 & 0 \\
0 & {\left[b_{2}\right]_{1}} & 0 & {\left[b_{5}\right]_{1}} & 0 & {\left[b_{1}\right]_{1}} & {\left[b_{7}\right]_{1}} \\
0 & {\left[c_{6}\right]_{1}} & {\left[c_{8}\right]_{1}} & {\left[c_{3}\right]_{1}} & 0 & {\left[c_{5}\right]_{1}} & {\left[c_{1}\right]_{1}}
\end{array}\right] \\
& A_{J}=\left[\begin{array}{ccccccc}
d & 0 & 0 & 1 & 0 & 0 & 0 \\
-1 & 0 & 0 & 0 & d & 0 & 0 \\
0 & -1 & 0 & 0 & 0 & d & 0 \\
0 & 0 & -1 & 0 & 0 & 0 & d \\
{\left[a_{6}\right]_{J}} & 0 & 0 & {\left[a_{3}\right]_{J}} & {\left[a_{1}\right]_{J}} & 0 & 0 \\
0 & \left(b_{4}\right)_{J} & 0 & {\left[b_{5}\right]_{J}} & 0 & {\left[b_{1}\right]_{J}} & {[b 7]_{J}} \\
0 & 0 & 0 & {\left[c_{3}\right]_{J}} & 0 & {\left[c_{5}\right]_{J}} & {\left[c_{1}\right]_{J}}
\end{array}\right] \\
& B_{J}=\left[\begin{array}{ccccccc}
0 & 0 & 0 & -1 & 0 & 0 & 0 \\
0 & 0 & 0 & 0 & d & 0 & 0 \\
0 & 0 & 0 & 0 & 0 & d & 0 \\
0 & 0 & 0 & 0 & 0 & 0 & d \\
0 & 0 & 0 & {\left[a_{4}\right]_{J}} & {\left[a_{2}\right]_{J}} & 0 & 0 \\
0 & 0 & 0 & {\left[b_{6}\right]_{J}} & 0 & {\left[b_{2}\right]_{J}} & {\left[b_{8}\right]_{J}} \\
0 & 0 & 0 & {\left[c_{4}\right]_{J}} & 0 & {\left[c_{6}\right]_{J}} & {\left[c_{2}\right]_{J}}
\end{array}\right] C_{J}=\left[\begin{array}{ccccccc}
d & 0 & 0 & 0 & 0 & 0 & 0 \\
1 & 0 & 0 & 0 & 0 & 0 & 0 \\
0 & 1 & 0 & 0 & 0 & 0 & 0 \\
0 & 0 & 1 & 0 & 0 & 0 & 0 \\
{\left[a_{5}\right]_{J}} & 0 & 0 & 0 & 0 & 0 & 0 \\
0 & {\left[b_{3}\right]_{J}} & 0 & 0 & 0 & 0 & 0 \\
0 & 0 & {\left[c_{7}\right]_{J}} & 0 & 0 & 0 & 0
\end{array}\right] r_{J}=\left[\begin{array}{l}
{\left[r_{1}\right]_{J}} \\
{\left[r_{2}\right]_{J}} \\
{\left[r_{3}\right]_{J}} \\
{\left[r_{4}\right]_{J}} \\
{\left[r_{5}\right]_{J}} \\
{\left[r_{6}\right]_{J}} \\
{\left[r_{7}\right]_{J}}
\end{array}\right]
\end{aligned}
$$

The tridiagonal system of equations is solved using the block tridiagonal elimination method. These computations are reiterated until the convergence criteria are convinced. Calculations are stopped $\left|\delta g^{(i)}\right|<\varepsilon$ with small, prescribed value $\varepsilon=0.01$. To confirm the technique's effectiveness, the skin friction values (Table 1) are matched with the previous publications. The outcomes are in accordance with existing literature. Also, temperature and concentration gradients are calculated for different values of heat source parameter and thermophoresis parameter local Nusselt number, and Sherwood numbers are calculated (Tables 2 and 3).

Table 1

Comparison of $-f$ " (0) for various values of $M, \alpha$

\begin{tabular}{lcccc}
\hline$\alpha$ & M & Fang et al. (2012) & Madaki et al. (2017) & Present results \\
\hline 0.25 & 0.5 & 0.93380 & 0.92461 & 0.95576 \\
& 1.0 & 1.00000 & 1.00000 & 1.00001 \\
& 5.0 & 1.11860 & 1.12623 & 1.08314 \\
0.5 & 0.5 & 0.97990 & 0.96335 & 0.99724 \\
& 1.0 & 1.00000 & 1.00000 & 1.00001 \\
& 5.0 & 1.02340 & 1.03339 & 1.01987 \\
\hline
\end{tabular}


Table 2

Values of $-\theta^{\prime}(0),-\phi^{\prime}(0)$ and $N u$, Sh for Pfr $=6.2, R d=0.3, m=0.3, \alpha=0.3, M=0.3 \beta=\infty, L e=2, N b=$ $0.1, \varepsilon=0$

\begin{tabular}{ccccc}
\hline$\lambda$ & $-\boldsymbol{\theta}^{\prime}(0)$ & $-\boldsymbol{\phi}^{\prime}(0)$ & $\mathrm{Nu}$ & Sh \\
\hline-0.5 & -2.68098 & 1.11043 & 1642 & -670 \\
-0.3 & -2.36495 & 0.81090 & 1448 & -497 \\
0 & -1.80102 & 0.28056 & 1103 & -172 \\
0.3 & -1.02767 & -0.43687 & 629 & 268 \\
0.5 & -0.19863 & -1.19258 & 122 & 730 \\
\hline
\end{tabular}

Table 3

Values of $-\theta^{\prime}(0)-\phi^{\prime}(0)$ and $N u$, Sh for $P r=6.2, R d=0.3, m=0.3, \alpha=0.3, M=0.3, \beta=\infty, L e=2, N b=0.1, \varepsilon=0$

\begin{tabular}{ccccc}
\hline $\mathrm{Nt}$ & $-\boldsymbol{\theta}^{\prime}(0)$ & $-\boldsymbol{\phi}^{\prime}(0)$ & $\mathrm{Nu}$ & $\mathrm{Sh}$ \\
\hline 0.1 & -1.57612 & 0.07069 & 965 & -43 \\
0.3 & -1.16806 & 1.32087 & 715 & -809 \\
0.5 & -0.85750 & 1.58877 & 525 & -973 \\
\hline
\end{tabular}

\section{RESULTS AND DISCUSSION}

The velocity, temperature, and concentration visual representations are plotted for various parameters shape parameter $(\mathrm{m})$, thickness parameter $(\alpha)$, adaptive thermal conductivity parameter $(\varepsilon)$, magnetic field parameter $(\mathrm{M})$, Casson parameter $(\beta)$, Brownian motion parameter $(\mathrm{Nb})$, thermophoresis parameter $(\mathrm{Nt})$ Chemical reaction parameter $(\gamma)$, porosity parameter $(\mathrm{k})$ are plotted using MATLAB.

Figures 1 and 2 signify velocity and concentration profiles for shape parameter $\mathrm{m}$; on increasing, shape parameter velocity and concentration profiles found to increase this is

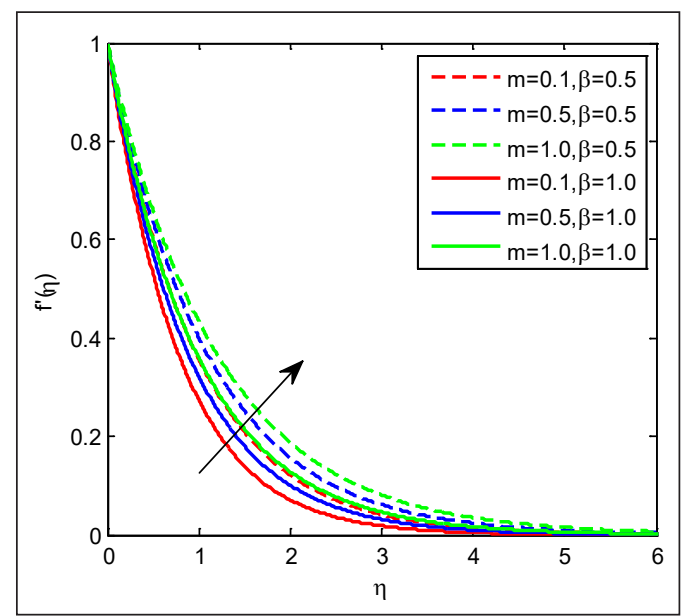

Figure 1. Velocity outlines form, $\beta$

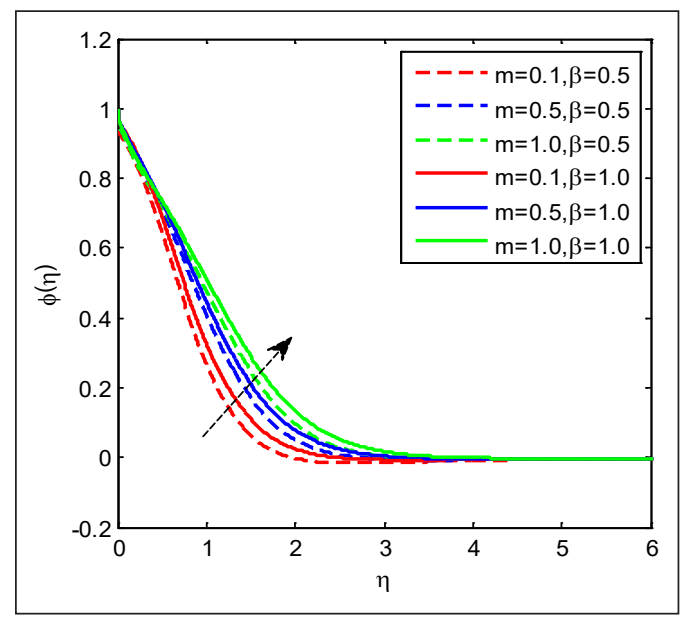

Figure 2. Concentration outlines form, $\beta$ 
MHD Casson Nano fluid across Porous Media on a Moving Plate

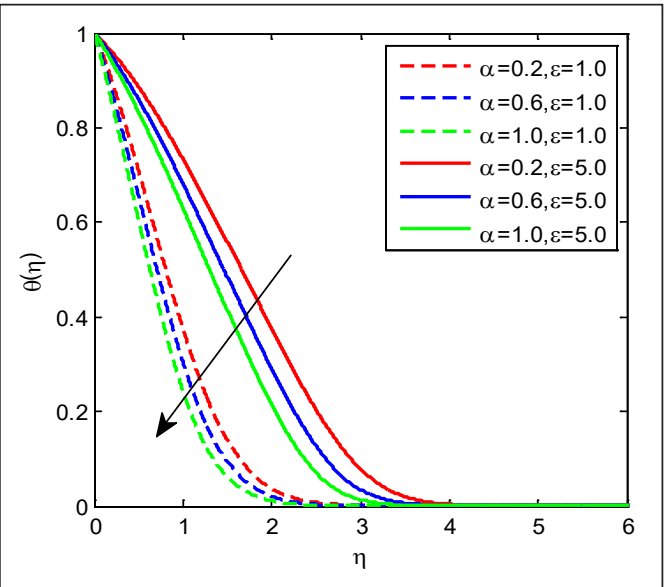

Figure 3. Temperature outlines for $\alpha, \varepsilon$

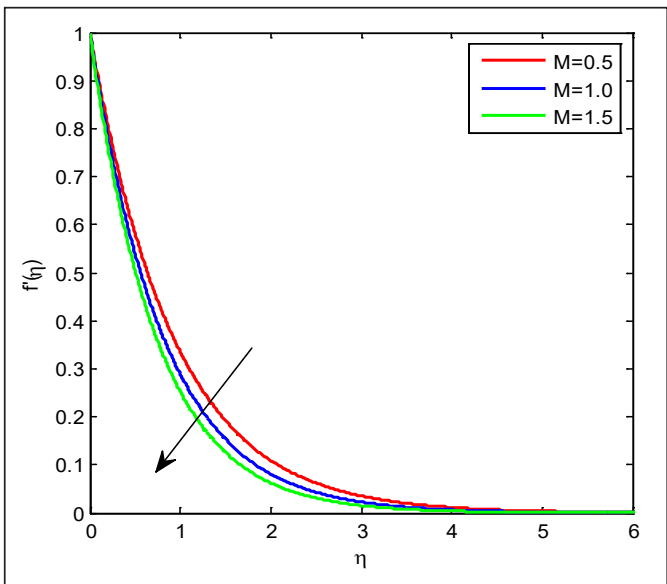

Figure 5. Velocity outlines for $\mathrm{M}$

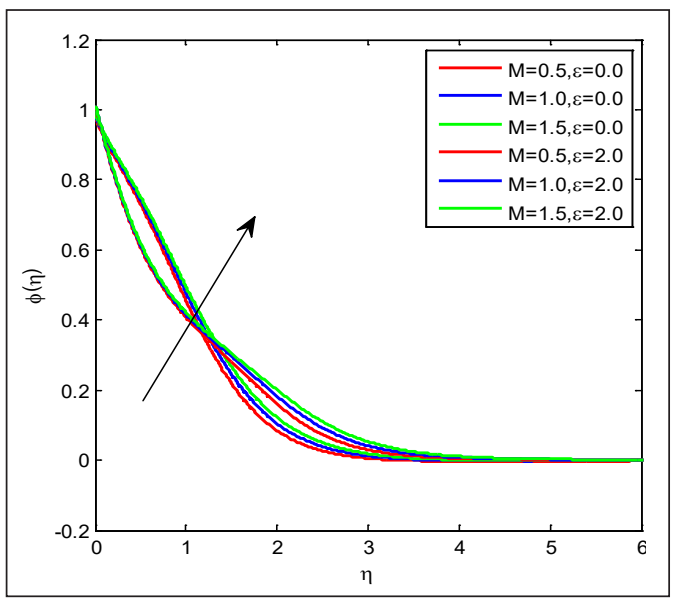

Figure 7. Concentration outlines for M, $\varepsilon$

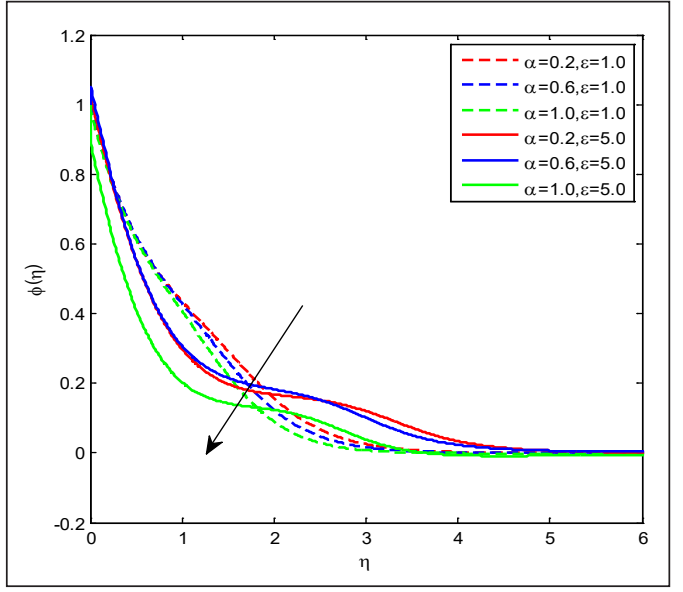

Figure 4. Concentration outlines for $\alpha, \varepsilon$

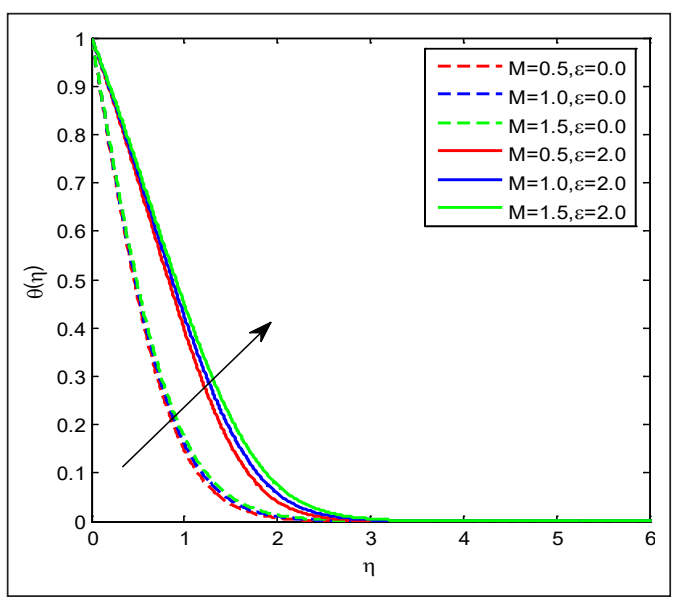

Figure 6. Temperature outlines for $\mathrm{M}, \varepsilon$

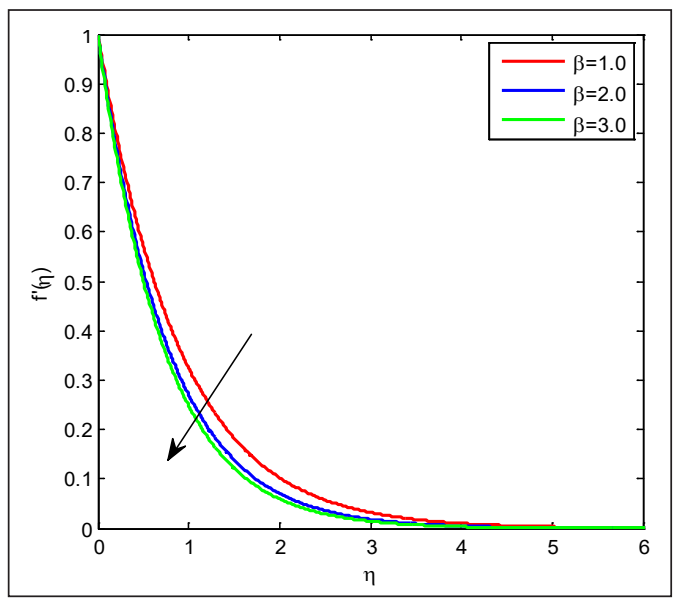

Figure 8. Velocity outlines for $\beta$ 


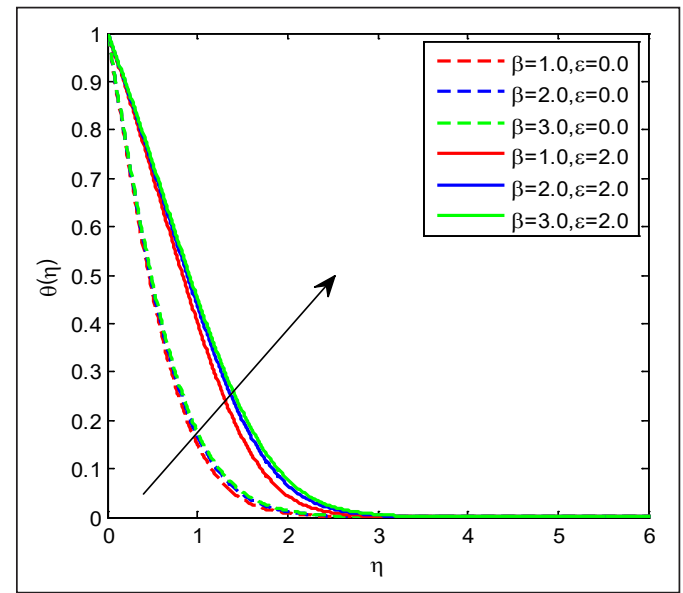

Figure 9. Temperature outlines for $\beta, \varepsilon$

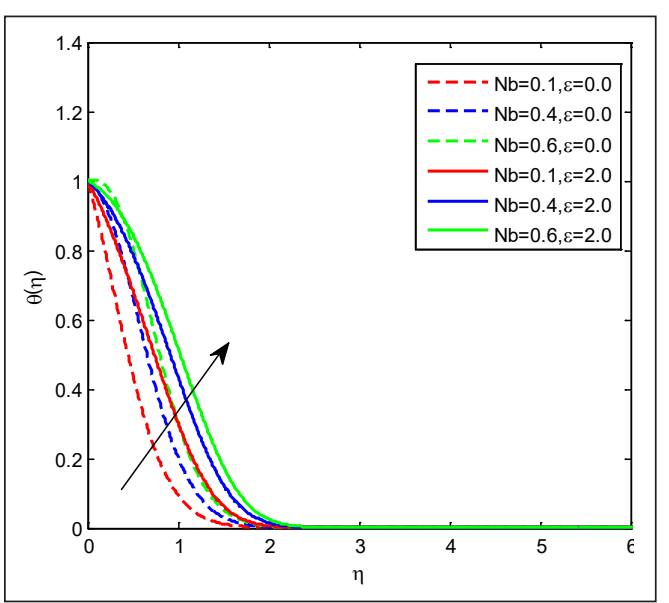

Figure 11. Temperature outlines for $\mathrm{Nb}, \varepsilon$

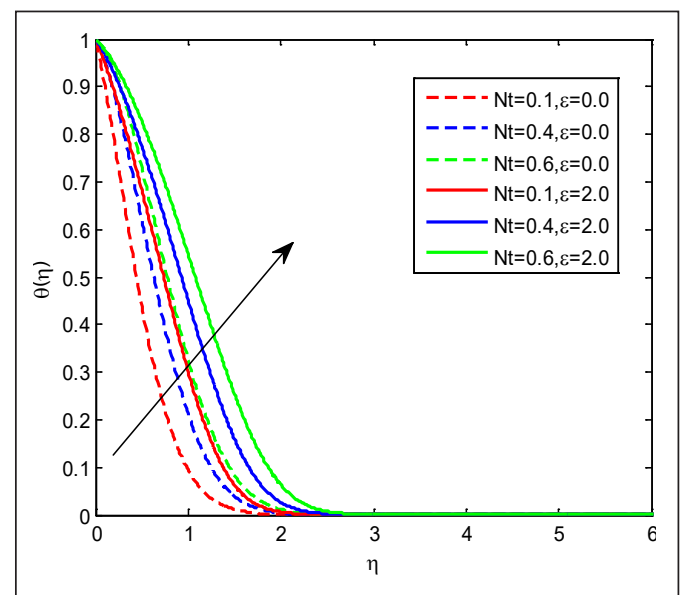

Figure 13. Temperature outlines for $\mathrm{Nt}, \varepsilon$

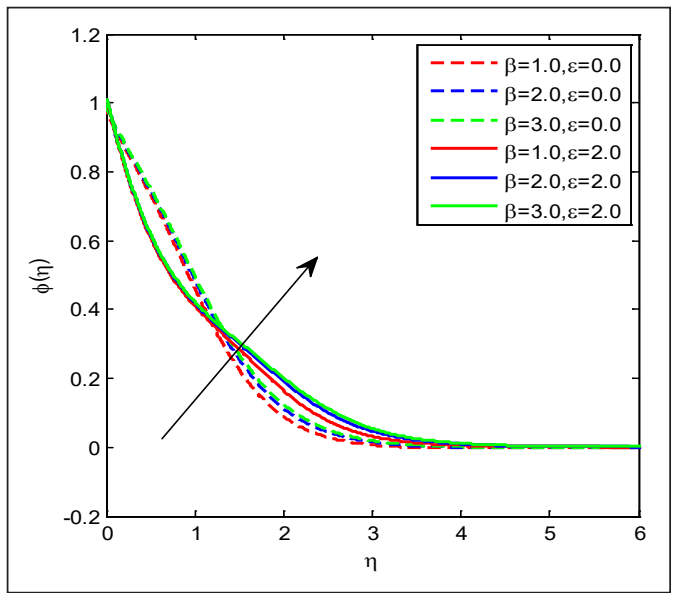

Figure 10. Concentration outlines for $\beta, \varepsilon$

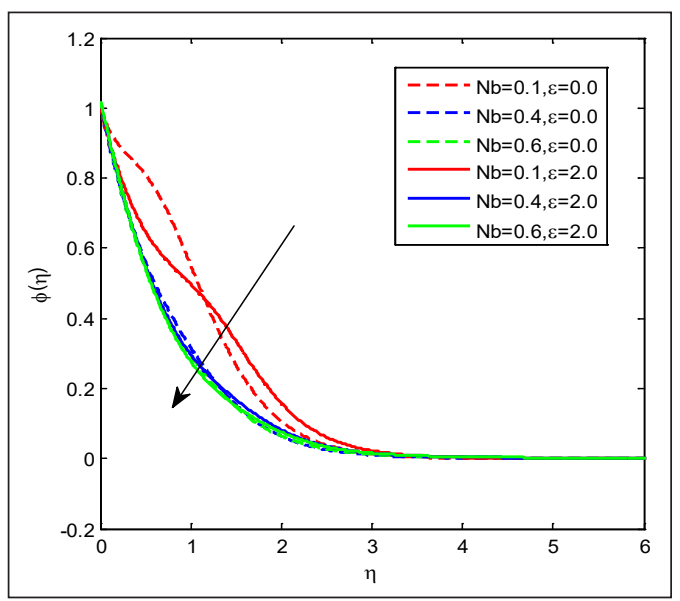

Figure 12. Concentration outlines for $\mathrm{Nb}, \varepsilon$

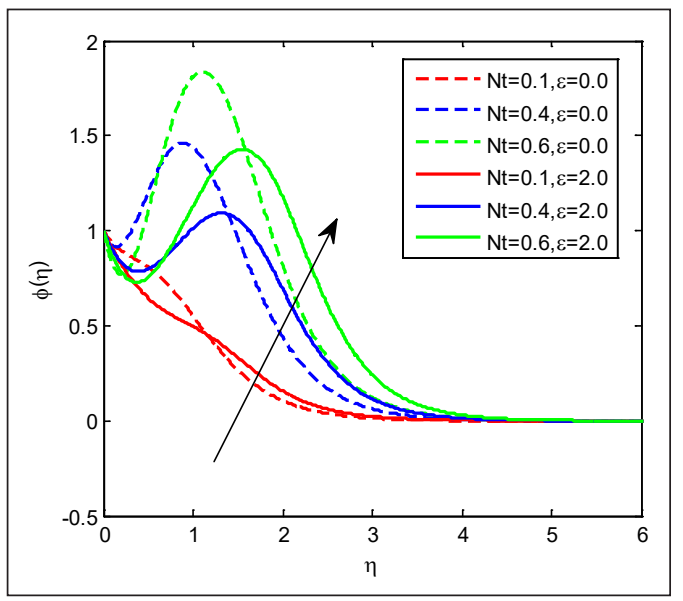

Figure 14. Concentration outlines for $\mathrm{Nt}, \varepsilon$ 
MHD Casson Nano fluid across Porous Media on a Moving Plate

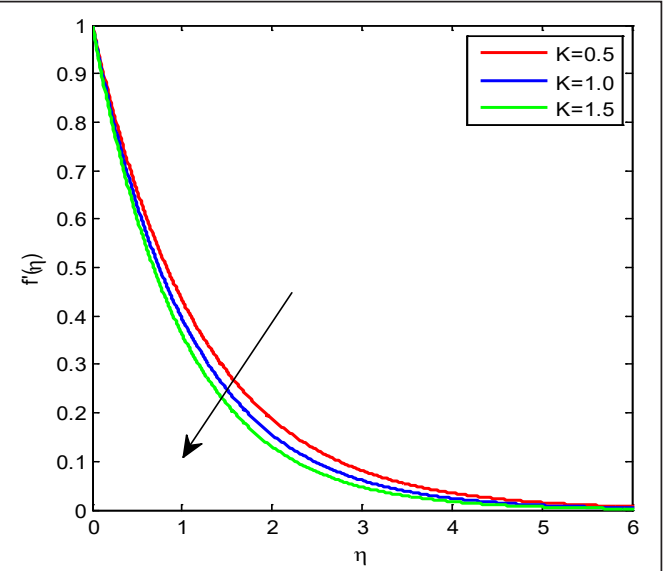

Figure 15. Velocity outlines for K

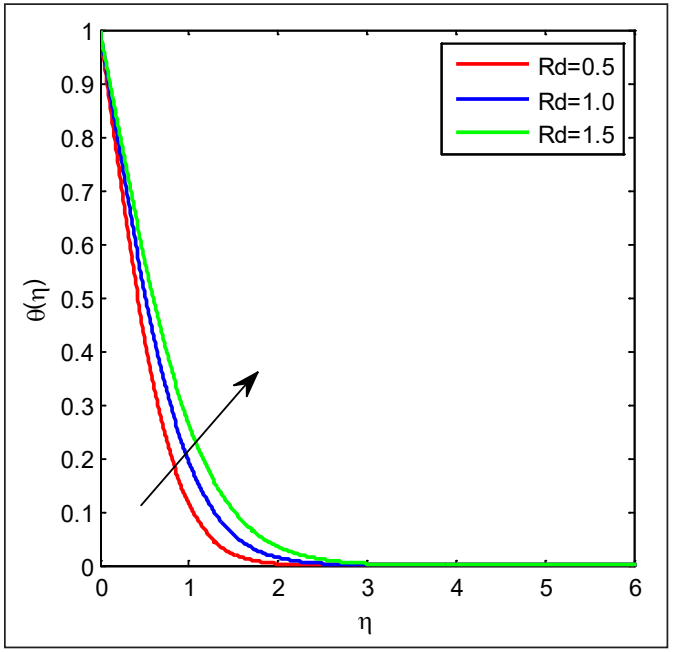

Figure 17. Temperature outlines for $\mathrm{Rd}$

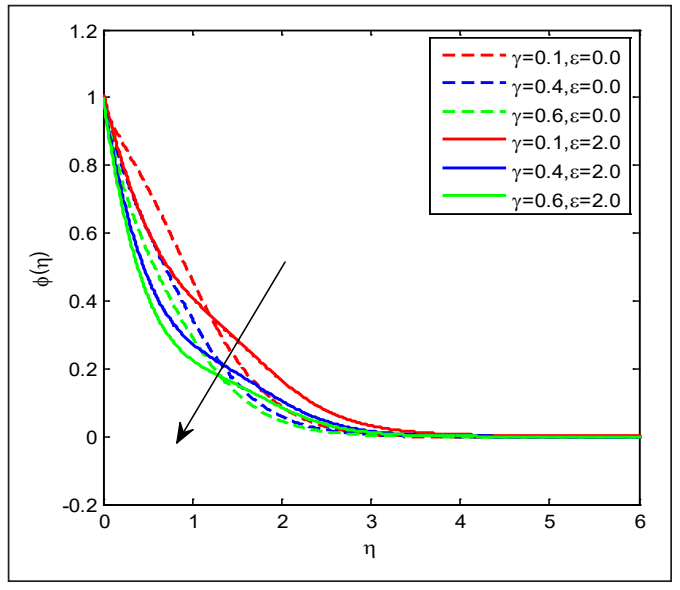

Figure 16. Concentration outlines for $\gamma$

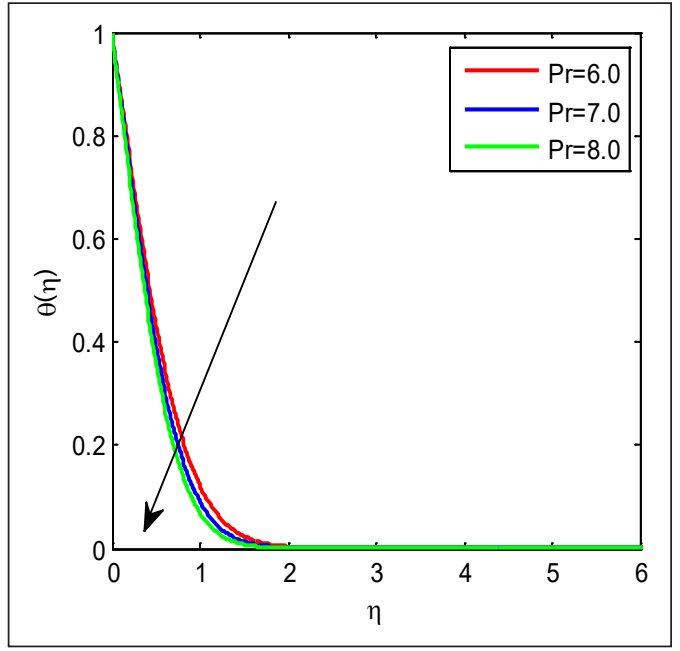

Figure 18. Temperature outlines for $\mathrm{Pr}$

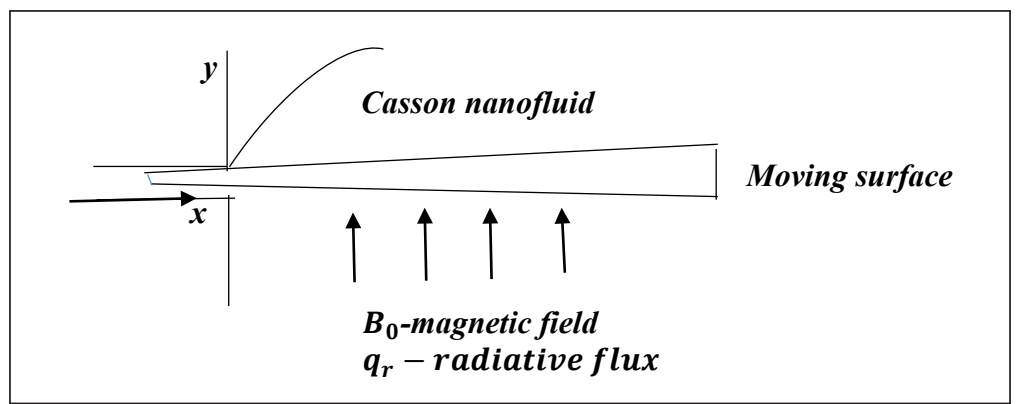

Figure 19. Flow model of the form 


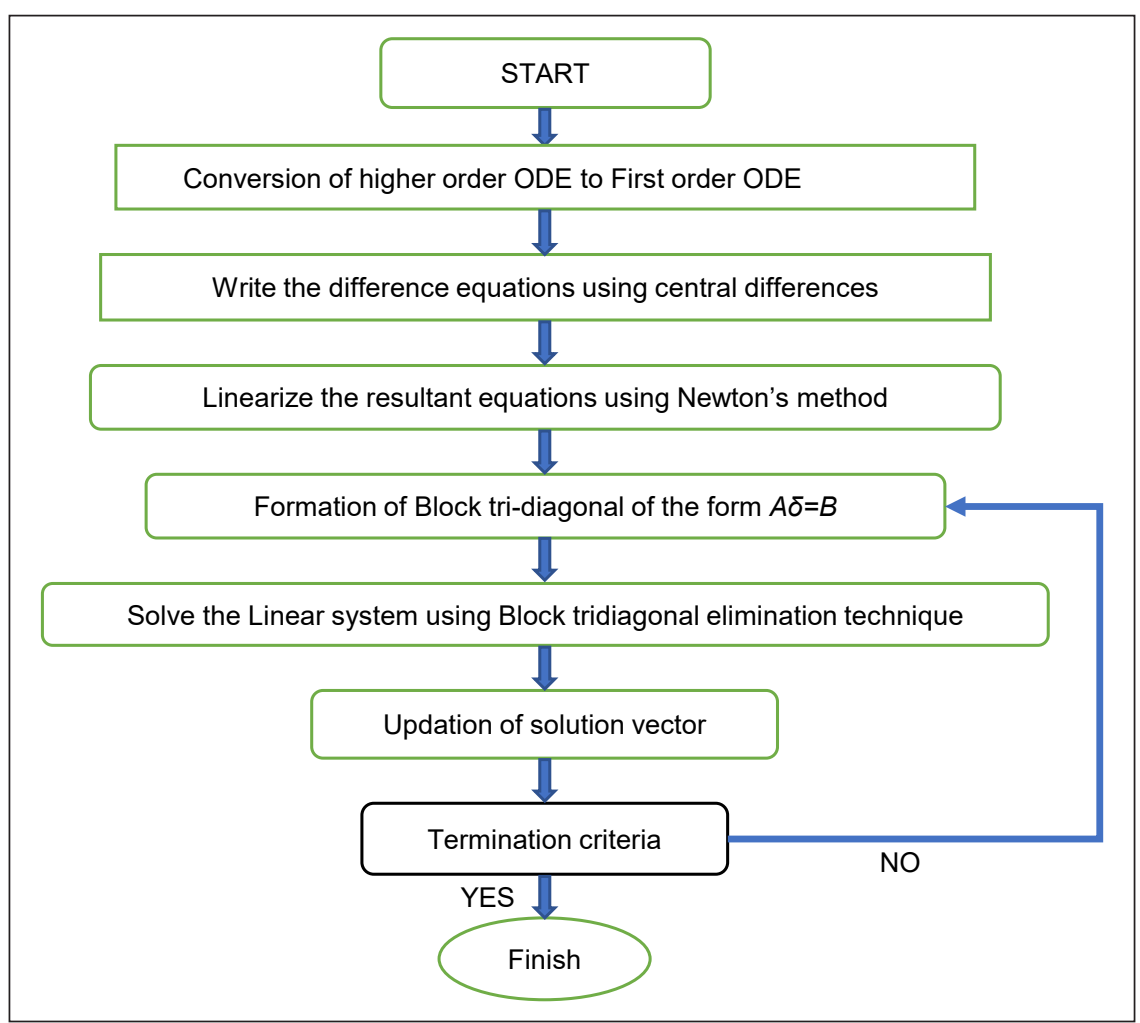

Figure 20. Flow chart of Keller box method

due to increasing values of $\mathrm{m}$ momentum and width of the thermal limit reduces. Also, the velocity of Casson fluid is high when compared with regular nanofluid. An opposite trend is observed in the case of concentration profiles. Figures 3 and 4 corresponds to temperature and concentration profiles for wall width parameter $\alpha$, and it is witnessed that for gradual values of wall width parameter, temperature and concentration shows fall tendency this is due to greater values of wall width parameter boundary layer becomes thinner. Also, the influence of variable thermal conductivity studied up variation is observed near the boundary layer in concentration profiles. Figure 5 represents velocity profiles for M. It is perceived that on increasing $M$ values, the velocity profile shows decreasing nature because of opposing force named as Lorentz force. Figures 6 and 7 portray thermal and concentration outlines for $\mathrm{M}, \varepsilon$. On intensifying magnetic parameter values, thermal and concentration outlines rose. Also, the influence of thermal conductivity parameter concentration profile shows decreasing nature before $\eta<1$ and opposite nature observed after $\eta>1$. Figure 8 shows velocity profiles for $\beta$. Increasing Casson parameter appears velocity profiles decline due to an increase in the value of plastic dynamic viscosity, which causes resistance in fluid flow. 
Figures 9 and 10 describe graphs of temperature and concentration for $\beta, \varepsilon$. It shows increasing behavior. Also, it is witnessed that variation in heat conductivity causes a rise in the thermal and concentration profiles of the fluid. Figures 11 and 12 represent thermal concentration profiles for $\mathrm{Nb}$. For progressive values of $\mathrm{Nb}$ temperature found to increase and concentration profile exhibits decreasing nature. The increase in Brownian motion leads to the movement of particles in a fluid. Hence, activating the movement of particles increases the heat produced, raising the temperature and decreasing the concentration. It is observed that the constant thermal conductivity concentration of molecules urns to a maximum near the surface, and thus, a maximum level will turn to a flat level with variable thermal conductivity. Figures 13 and 14 represent temperature and concentration outlines of Nt. For higher observations of Nt, it guides to increase in temperature, concentration outlines this is because of the force of the thermophoresis was produced by the surface amount of mass relocation builds a far slippery flow on the non-linear elongating surface, hence, with much quantities of thermophoresis the fluid is an extra warm move away across the surface, and because of the thermophoresis force presence the flow is fast from the non-linear surface, and that is lead to increasing the nanoparticle concentration boundary-layer. For growing values of thermal conductivity, concentration was found to increase. Figure 15 represents velocity profiles for porosity parameters. The growth in the absorbent parameter of the liquid enhances the viscosity of the fluid or falls in the elongating rate of the expediting surface, leading to a step-by-step reduction in the velocity of the fluid. Figure 16 represents concentration profiles for chemical reaction parameters. An escalation in chemical reaction values will restrain the concentration of the fluid-greater observations of $\gamma$ aggregates to a drop in the chemical molecular circulation. Figure 17 portrays temperature profiles of radiation parameters that will deliver more heat to the fluid, resulting in a temperature rise. Figure 18 represents temperature profiles for the Prandtl number. On enhancing the Prandtl number, the heat conductivity of the fluid drops, which causes declination in thermal profiles. Figure 19 represents flow model of the problem while Figure 20 represents flow chart of the Keller box method.

\section{CONCLUSIONS}

In the present investigation, the impact of chemical reaction on magnetohydrodynamic boundary layer of Casson nanofluid flow through permeable media above a moving plate adaptable thickness is studied. As a result, the following results are obtained.

- Velocity profile increases for shape parameter and decreases for the magnetic parameter, Casson parameter, porosity parameter.

- Temperature profile found to decrease wall thickness parameter, Prandtl number. Increases for the magnetic parameter, Casson parameter, Brownian movement parameter, thermophoresis parameter, radiation parameter. 
- Concentration profiles were found to increase for shape parameter at various observations of Casson parameter, increases for the magnetic parameter for $\eta>1$, and decreases for $\eta<1$.Decreases for wall thickness, Brownian movement, chemical reaction parameters, increases for thermophoresis parameter at different thermal conductivity values.

\section{ACKNOWLEDGEMENT}

The authors are thankful to Koneru Lakshmaiah Education Foundation, Guntur, India for providing research facilities to complete this project.

\section{REFERENCES}

Abdel-wahed, M. S., Elbashbeshy, E. M. A., \& Emam, T. G. (2015). Flow and heat transfer over a moving surface with non-linear velocity and variable thickness in a nanofluids in the presence of Brownian motion. Applied Mathematics and Computation, 254, 49-62. https://doi.org/10.1016/j.amc.2014.12.087

Ahmad, K., Hanouf, Z., \& Ishak, A. (2017). MHD Casson nanofluid flow past a wedge with Newtonian heating. The European Physical Journal Plus, 132(2), 1-11. https://doi.org/10.1140/epjp/i2017-11356-5

Beg, O. A., Thumma, T., \& Sheri, S. R. (2009). Finite element computation of magnetohydrodynamic nanofluid. Computational Materials Science, 46(4), 1028-1037.

Besthapu, P., \& Bandari, S. (2015). Mixed convection MHD flow of a Casson nanofluid over a nonlinear permeable stretching sheet with viscous dissipation. Journal of Applied Mathematics and Physics, 03(12), 1580-1593. https://doi.org/10.4236/jamp.2015.312182

Biswas, R., \& Ahmmed, S. F. (2018). Effects of hall current and chemical reaction on Magnetohydrodynamics unsteady heat and mass transfer of Casson nanofluid flow through a vertical plate. Journal of Heat Transfer, 140(9), 1-12. https://doi.org/10.1115/1.4039909

Choi, S. U. S. (2008). Nanofluids: A new field of scientific research and innovative applications. Heat Transfer Engineering, 29(5), 429-431. https://doi.org/10.1080/01457630701850778

Das, S. K., Choi, S. U. S., Yu, W., \& Pradeep, T. (2007). Nanofluids. John Wiley \& Sons. https://doi. org/10.1002/9780470180693

Dharmaiah, G., Chamkha, A. J., Vedavathi, N., \& Balamurugan, K. S. (2019a). Viscous dissipation effect on transient aligned magnetic free convective flow past an inclined moving plate. Frontiers in Heat and Mass Transfer, 12, Article 17. https://doi.org/10.5098/hmt.12.17

Dharmaiah, G., Vedavathi, N., Balamurugan, K. S., \& Ramakrishna, K. (2019b). A study on MHD boundary layer flow rotating frame nanofluid with chemical reaction. Frontiers in Heat and Mass Transfer, 12, Article 10. https://doi.org/10.5098/hmt.12.10

Dharmaiah, G., Sridhar, W., Balamurugan, K. S., \& Kala, K. C. (2020). Hall and ion slip impact on magnetotitanium alloy nanoliquid with diffusion thermo and radiation absorption. International Journal of Ambient Energy, 1-11. https://doi.org/10.1080/01430750.2020.1831597 
Dharmaiah, G., Vedavathi, N., Balamurugan, K. S., \& Prakash, J. (2017). Heat transfer on MHD nanofluid flow over a semi infinite flat plate embedded in a porous medium with radiation absorption, heat source and diffusion thermo effect. Frontiers in Heat and Mass Transfer, 9, Article 38. https://doi.org/10.5098/hmt.9.38

Fang, T., Zhang, J., \& Zhong, Y. (2012). Boundary layer flow over a stretching sheet with variable thickness. Applied Mathematics and Computation, 218(13), 7241-7252. https://doi.org/10.1016/j.amc.2011.12.094

Ibrahim, W., \& Makinde, O. D. (2016). Magnetohydrodynamic stagnation point flow and heat transfer of Casson nanofluid past a stretching sheet with slip and convective boundary condition. Journal of Aerospace Engineering, 29(2), Article 04015037. https://doi.org/10.1061/(asce)as.1943-5525.0000529

Ismail, H., Abdel-Wahed, M., \& Omama, M. (2021). Effect of variable thermal conductivity on the MHD boundary-layer of Casson-nanofluid over a moving plate with variable thickness. Thermal Science, 25(1 Part A), 145-157. https://doi.org/10.2298/tsci190324293i

Khan, M., Shahid, A., Salahuddin, T., Malik, M. Y., \& Mushtaq, M. (2018). Heat and mass diffusions for Casson nanofluid flow over a stretching surface with variable viscosity and convective boundary conditions. Journal of the Brazilian Society of Mechanical Sciences and Engineering, 40(11), 1-10. https://doi. org/10.1007/s40430-018-1415-y

Konda, J. R., Madhusudhana, N. P., \& Konijeti, R. (2018). MHD mixed convection flow of radiating and chemically reactive Casson nanofluid over a nonlinear permeable stretching sheet with viscous dissipation and heat source. Multidiscipline Modeling in Materials and Structures, 14(3), 609-630. https://doi. org/10.1108/mmms-10-2017-0127

Madaki, A. G., Roslan, R., Kandasamy, R., \& Chowdhury, M. S. H. (2017). Flow and heat transfer of nanofluid over a stretching sheet with non-linear velocity in the presence of thermal radiation and chemical reaction. In AIP Conference Proceedings (Vol. 1830, No. 1, p. 020014). AIP Publishing LLC. https:// doi.org/10.1063/1.4980877

Mahdy, A., \& Chamkha, A. (2015). Heat transfer and fluid flow of a non-Newtonian nanofluid over an unsteady contracting cylinder employing Buongiorno's model. International Journal of Numerical Methods for Heat \& Fluid Flow, 25(4), 703-723. https://doi.org/10.1108/hff-04-2014-0093

Mahmood, A., Jamshed, W., \& Aziz, A. (2018). Entropy and heat transfer analysis using Cattaneo-Christov heat flux model for a boundary layer flow of Casson nanofluid. Results in Physics, 10, 640-649. https:// doi.org/10.1016/j.rinp.2018.07.005

Malik, M. Y., Naseer, M., Nadeem, S., \& Rehman, A. (2014). The boundary layer flow of Casson nanofluid over a vertical exponentially stretching cylinder. Applied Nanoscience, 4(7), 869-873. https://doi.org/10.1007/ s13204-013-0267-0

Nagalakshm, P. S. S., \& Vijaya, N. (2020). MHD flow of Carreau nanofluis explored using CNT over a nonlinear stretched sheet. Frontiers in Heat and Mass Transfer, 14(4), 1-9. https://doi.org/10.5098/hmt.14.4

Oyelakin, I. S., Mondal, S., \& Sibanda, P. (2016). Unsteady Casson nanofluid flow over a stretching sheet with thermal radiation, convective and slip boundary conditions. Alexandria Engineering Journal, 55(2), 1025-1035. https://doi.org/10.1016/j.aej.2016.03.003 
Reddy C, S., Naikoti, K., \& Rashidi, M. M. (2017). MHD flow and heat transfer characteristics of Williamson nanofluid over a stretching sheet with variable thickness and variable thermal conductivity. Transactions of A. Razmadze Mathematical Institute, 171(2), 195-211. https://doi.org/10.1016/j.trmi.2017.02.004

Reddy, J. V. R., Sugunamma, V., \& Sandeep, N. (2018). Slip flow of MHD Casson-nanofluid past a variable thickness sheet with joule heating and viscous dissipation: A comparative study using three base fluids. Journal of Nanofluids, 7(6), 1113-1121. https://doi.org/10.1166/jon.2018.1540

Sheikholeslami, M., Abelman, S., \& Ganji, D. D. (2014). Numerical simulation of MHD nanofluid flow and heat transfer considering viscous dissipation. International Journal of Heat and Mass Transfer, 79, 212222. https://doi.org/10.1016/j.ijheatmasstransfer.2014.08.004

Singh, A. (2008). Thermal conductivity of nanofluids. Defence Science Journal, 58(5), 600-607. https://doi. org/10.14429/dsj.58.1682

Sridhar, W., \& Lakshmi, G. V. (2020). MHD nanofluid over a stretching surface immersed in double stratified media with radiation and partial slip conditions. International Journal of Advanced Science and Technology, 29(9s), 3204-3213.

Talla, H., Kumari, P., \& Sridhar, W. (2018). Numerical study to diffusion of chemically reactive species over MHD exponentially stretching surface of a Casson fluid. International Journal of Mechanical Engineering and Technology, 9(10), 470-481.

Uddin, M. J., Bég, O. A., \& Ismail, A. I. (2015). Radiative convective nanofluid flow past a stretching/ shrinking sheet with slip effects. Journal of Thermophysics and Heat Transfer, 29(3), 513-523. https:// doi.org/10.2514/1.t4372

Vijaya, N., Babu, S. G., \& Lakshmi, V. N. (2020). Influence of critical parameters on liquid thin film flow of Casson nanofluid over elongated sheet under thermoporesis and Brownian motion. Frontiers in Heat and Mass Transfer, 15, Article 23. https://doi.org/10.5098/hmt.15.23

Wang, X. Q., \& Mujumdar, A. S. (2008a). A review on nanofluids - part I: Theoretical and numerical investigations. Brazilian Journal of Chemical Engineering, 25(4), 613-630. https://doi.org/10.1590/ s0104-66322008000400001

Wang, X. Q., \& Mujumdar, A. S. (2008b). A review on nanofluids - part II: Experiments and applications. Brazilian Journal of Chemical Engineering, 25(4), 631-648. https://doi.org/10.1590/s010466322008000400002 


\section{List of Symbols}

\begin{tabular}{|c|c|c|c|}
\hline Symbol & Description & Unit & Dimension \\
\hline v & Kinematic viscosity & $m^{2} s^{-1}$ & $L^{2} T^{11}$ \\
\hline$\sigma$ & Electrical conductivity & $s / m$ & $M^{-1} L^{-2} T^{3} A^{2}$ \\
\hline$\rho$ & Base fluid density & $\mathrm{kg} / \mathrm{m}^{3}$ & $M L^{-3}$ \\
\hline$\alpha$ & Thermal diffusion & $m^{2} s^{-1}$ & $M L T^{-3} K^{-1}$ \\
\hline$C_{p}$ & Heat capacity of fluid at constant pressure & $J . \mathrm{kg}^{-1} \mathrm{~s}^{-1}$ & $L^{2} T^{-2} K^{-1}$ \\
\hline$\varepsilon$ & Thermal conductivity & $W / m \cdot k$ & $M L T^{-3} K^{-1}$ \\
\hline $\mathrm{D}_{\mathrm{T}}$ & Thermo-diffusion coefficient & $m^{2} / s$ & \\
\hline $\mathrm{D}_{\mathrm{B}}$ & Brownian diffusion coefficient & $m^{2} / s$ & \\
\hline $\mathrm{u}$ & Velocity component in $\mathrm{x}$-axis direction & & \\
\hline $\mathrm{v}$ & Velocity component in y-axis direction & & \\
\hline$\beta$ & Casson parameter & & \\
\hline$\tau$ & Ratio of nano particle heat capacity and the fluid heat capacity & & \\
\hline B & Magnetic field & & \\
\hline Q & Heat source parameter & & \\
\hline$a, b$ & Constants & & \\
\hline$T_{w}{ }^{*}$ & Surface temperature & & \\
\hline$T_{\infty}$ & Ambient temperature & & \\
\hline $\mathrm{m}$ & Shape parameter $(>-1)$ & & \\
\hline$\varphi$ & Stream function & & \\
\hline M & Magnetic field parameter & & \\
\hline $\operatorname{Pr}$ & Prandtl number & & \\
\hline $\mathrm{Nb}$ & Brownian motion parameter & & \\
\hline $\mathrm{Nt}$ & Thermophoresis parameter & & \\
\hline$\lambda$ & Heat source parameter & & \\
\hline $\mathrm{Sc}$ & Schmidt number & & \\
\hline$q_{r}$ & Radiative heat flux & & \\
\hline
\end{tabular}


